\title{
The influence of privacy dispositions on perceptions of information transparency and personalization preferences
}

\author{
Sabrina Hauff \\ University of Augsburg \\ sabrina.hauff@wiwi.uni- \\ augsburg.de
}

\author{
Olga Dytynko \\ University of Augsburg \\ olga.dytynko@student.uni- \\ augsburg.de
}

\author{
Daniel Veit \\ University of Augsburg \\ veit@wiwi.uni-augsburg.de
}

\begin{abstract}
To attract customers, firms offer personalized services. This is perceived beneficial by many customers as it enhances the purchase experience and addresses customers' needs. However, to offer personalized services, customer data has to be collected and analyzed. This practice gives rise to privacy concerns and can inhibit the usage of such services. Our research aims to address the tension between personalization and privacy by applying information boundary theory to investigate how respondents' disposition to value privacy and the availability of information transparency features influences individuals' intention to disclose information to personalized services. Based on an experimental study, we find a significant interaction between disposition to value privacy and personalization, while the implementation of transparency features does not yield substantial changes in information disclosure. Thus, in order to successfully offer personalized services, we recommend that practitioners take individuals' privacy preferences into account for their service design.
\end{abstract}

\section{Introduction}

The personalization of products and services allows digital businesses to deliver additional value to customers by better targeting their needs and interests. Thus, personalization is a potential source of competitive advantage [1]. To offer personalized products and services, marketers worldwide collect and analyze identity-related customer data such as email addresses, location, demographics, or lifestyle details [2]. However, customers may view such organizational practice as an invasion of their information privacy, as they have to give up personal information to businesses in exchange for promised benefits [3]. According to the latest TRUSTe Privacy Index 2016, $92 \%$ of U.S. internet users have privacy-related concerns while $45 \%$ of respondents are more worried about their online privacy than they were one year ago [4]. Therefore, customers have to trade off the benefits of using personalized services with the associated risks $[5,6]$.

This evaluation of benefits and risks depends on people's privacy valuation and differs between individuals [7,8]. However, to the best of our knowledge, the impact of a general valuation of privacy on information disclosure has not been studied before. As the offering of personalized products and services is highly dependent on customer information, the prevalent study addresses this research gap.

Surveys continuously find that consumers wish to have more transparency about personal data collection and usage [4]. Yet, Awad and Krishnan revealed the personalization-privacy paradox [9]: People who value transparency most have a low willingness to be profiled for personalization purposes. To overcome this paradox, they suggest the investigation of transparency features which may persuade consumers to partake in personalization [9]. Previous research highlights that the implementation of transparency enhancing mechanisms could be one way to overcome people's privacy concerns $[8,10]$ because it increases perceived procedural fairness and fosters reciprocity [11,12]. It also increases people's willingness to spend money on websites that communicate their privacy practices in an easily accessible and understandable way [13]. Yet, there might also be a contrary effect of transparency: If it is made explicit to customers how much information is collected and how it is used, it might not reduce but increase their fears. This increase might be in particular the case for people who highly value their privacy. Overall, we thus pose the following research question: How do individuals' disposition to value privacy, personalization of a service, and information transparency influence their willingness to disclose information?

The remainder of this paper first provides relevant theoretical background and the study hypotheses. This is followed by a description of our research 
methodology, namely an experimental setup. We then present our findings. In chapter five, we critically discuss them, provide implications for theory and practice, and offer avenues for future research. The paper ends with a conclusion of our study.

\section{Theoretical foundations and hypotheses development}

This section reviews prior research on the relationship between personalization, information transparency, and privacy. It also introduces the information boundary theory which is used as theoretical lens for our study.

\subsection{Prior research on personalization, information transparency, and privacy}

Personalization is "the ability to proactively tailor products and [...] purchasing experiences to tastes of individual consumers based upon their personal and preference information" [6:181]. It mostly appears in two forms: personalized advertising and personalized services such as product recommendations [9]. For both kinds of personalization firms need to build customer profiles based on data users provide voluntarily or information acquired through observation of users' online behavior $[6,14]$. In this paper we focus on personalized services.

IS researchers have investigated both benefits and risks of personalization. For instance, personalized services can reduce information overload and thereby increase users' satisfaction [15]. If personalization increases customers' sense of control and freedom, for example through personalized order tracking, purchase histories, or e-mail notification of new products and special deals, it will be appreciated by many customers. Furthermore, web personalization that includes self-referent and relevant content is valued by consumers as a decision aid because it reduces cognitive efforts in their decision making process [16].

However, some customers may refuse to use offered services, even if they value personalization, as they are often concerned about their information privacy due to potential commercial misuse of their personal data [6,9]. Information privacy reflects the extent to which individuals are able to control how, when, and what amount of personal information about them is revealed to others [17]. Privacy concerns are individual concerns related to opportunistic behavior with regard to personal data submitted over the internet. These concerns represent the degree to which individuals consider a potential privacy loss through the disclosure of personal information $[18,19]$. These customers" concerns arise as "personalization is not feasible without sharing personal information, and free allowance of services is not feasible without some exploitation of this information by the vendor" [14:196]. Thus, while some consumers are willing to sacrifice their privacy to some extent in exchange for obtained benefits (i.e. personalization), others protect their privacy as a fundamental right [20]. The resulting personalization-privacy trade-off suggests customers will likely use personalization services in case they deliver them a certain value that overrides existing privacy concerns [6,21]. Treiblmaier and Pollach further investigated this issue in an exploratory study and found that people's perception of the benefits and costs of personalized communication depends on their general attitude towards revealing personal data [8].

Thus, online customers' privacy preferences are heterogeneous and customers experience a trade-off between information disclosure benefits and privacy concerns in distinct ways [7,8]. One concept that depicts people's general privacy preferences in particular is disposition to value privacy (DTVP) $[22,23]$. DTVP is a personality attribute that represents a person's general need to preserve specific information boundaries in order to frame their personal space in different situations and contexts. Previous research found that DTVP influences individuals' assessment of privacy risks [22]. It follows that individuals who generally value privacy more might also perceive risks of information disclosure for personalization differently than people with a lower DTVP and that their resulting information disclosure behavior may vary, too. Yet, to the best of our knowledge, the impact of a general valuation of privacy on information disclosure for personalization offerings has not been studied before.

In order to reduce privacy concerns and thereby increase service usage, previous research has investigated transparency enhancing mechanisms amongst others [9,13,24]. Information transparency is the extent to which an online firm provides features that allow customers to access the data collected about them as well as informs them on how and for what purposes the acquired information is going to be used [9]. From a customer's perspective, privacy policies and information transparency features are not substitutes [9]: transparency features give for example an overview and thus enhanced sense of which information is collected and how it could be used by organizations in an accessible and understandable way. In combination, privacy policies and transparency features can facilitate the understanding of a company's data usage policy for customers. If such information is absent or if customers cannot easily get an understanding of them, customers will likely 
hesitate to share requested information [21]. In addition, Tsai et al. find that individuals are willing to pay more on websites that display privacy information in a more obvious and intuitive manner [13].

However, Awad and Krishnan also discovered the personalization-privacy paradox [9]: Customers who value information transparency features are less willing to be profiled for personalized offerings. They speculated that these people might be so-called privacy fundamentalists who put high emphasis on their privacy and are thus less willing to disclose information in general and for personalized offerings in particular. Nonetheless, they argued that high quality transparency might also be useful in persuading such privacy fundamentalists to disclose information. Yet, to the best of our knowledge, the usefulness of transparency mechanisms depending on individuals' DTVP has not been studied so far, but could be very interesting as it not only adds to our theoretical understanding but could also inform practitioners how to design personalized services.

In summary, current insights on the role of individuals' DTVP and information transparency features within a consumer's personalization-privacy dilemma are limited. In order to address this shortfall and improve the understanding of the trade-off between personalization and privacy, further research focusing on these aspects is needed and this paper aims at addressing this research gap.

\subsection{Information boundary theory}

A broad range of established theories such as expectancy-value theory, protection motivation theory or social response theory have been used as foundation in information privacy research [25]. This study draws on information boundary theory (IBT; also called communication privacy management theory) which explains how individuals decide what kind of personal information should be disclosed when and to whom $[22,26]$. The theory is suitable for our study due to several reasons. First of all, IBT considers interrelations between benefits and risks of information disclosure that have been discovered in the context of personalized services [5,21]. This differentiates IBT from the often used privacy calculus, in which risks and benefits are independently assessed and then weighted against each other [19,27]. Second, IBT illustrates the rise of an individual's privacy concerns depending on an individual's personality and related disposition to privacy $[22,25]$. Third, IBT explains how an individual's privacy concerns and the evaluation of associated risks depend on situational factors. Situational factors represent the extent of personalization and transparency offered to a customer.
As a result, IBT is an appropriate theoretical lens that enables us to address the defined research question.

IBT was developed by Petronio [26,28]. She leveraged the theory to explain the disclosure of personal information between partners in marital couples [28]. The theory has been adopted by IS researchers to study the formation of privacy concerns of website users [22] and to explain the tension between information disclosure and privacy in online commercial transactions [29], in social media [30], and in the mobile context [31].

According to IBT, three processes of boundary management take place: 1) Boundary rule formation, which refers to how individuals develop rules to manage their privacy based on the nature of the information to be shared, an individual's personality, environmental factors, and an interrelated risk-benefit assessment; 2) Boundary coordination, which refers to the management of a person's boundaries and comprises boundary permeability (how thin or thick the boundary is and what information is shared with whom), boundary linkage (the strength of the connection between the involved parties), and boundary ownership (reflects the responsibilities and rights regarding the spread of information); 3) Boundary turbulences, which arise if a person's boundaries are invaded and can lead to a reformulation of the boundary rules [26].

\subsection{Hypotheses development}

Offering personalized services can have two different effects: On the one hand, the extent of personalization can affect perceived benefits related to a service. For example, higher personalization is beneficial to a customer because the website's product and service offerings can be better matched to a customer's needs and preferences [32]. On the other hand, the extent of personalization also affects perceived privacy risks related to a service. As the customer has to share personal information in order to receive highly personalized services, personalization may provoke customers' privacy concerns by enhancing perceived risks associated with information disclosure [5,9,11,31]. Thus, higher personalization can provide more benefits to customers, but might at the same time trigger higher privacy concerns, as individuals perceive stronger information boundary penetration involved in the process of personalization.

Moreover, a differentiation based on people's DTVP can help to shed further light on the issue of risk and benefit perceptions: An individual with higher DTVP will more likely expect negative outcomes associated with the disclosure of personal information in a particular online setting such as the loss of privacy 
than someone with a lower DTVP [22]. If we compare the risk perceptions of customers with distinct DTVP levels by identical boundary penetration in a given situation (e.g. a particular personalized service offering and available privacy information), their perceptions of risks and benefits will not be the same, but people with high DTVP will have higher concerns and see less value $[9,23]$. Higher privacy concerns lead to higher perceived risks and stronger fears of intrusion, while the absence of meaningful benefits strengthens the privacy boundaries for information exchange in such scenarios. The opposite holds for low concerns and respectively weaker risk perceptions of those customers who value personalization [22].

Therefore, high personalization that requires businesses' extensive knowledge about customers is viewed as an intrusion to people's privacy boundaries according to IBT. In this case, people with high DTVP who in general have great worries with regard to their privacy are reluctant to share personal information with services in general, whereas people with low DTVP are more willing to trade their personal information, but only if substantial benefits of personalization are offered. Thus, we propose:

Hla: Individuals with low DTVP have higher intentions to disclose information to highly personalized services than for services with a low level of personalization.

H1b: Individuals with low DTVP have higher intentions to disclose information to highly personalized services than people with high DTVP.

To shed further light on the effect of transparency features as possibility to reduce the personalizationprivacy paradox, we differentiate between individuals with low and high DTVP in the following because they differently assess risks and benefits [22] which results in different boundary rules. Thus, transparency may have contrary effects on information disclosure depending on not only individuals' DTVP, but also on their level of awareness of the collection and usage of information being asked for.

Following IBT, individuals with low DTVP have in general a lower tendency to preserve their private information space and are thus more willing to share information [22]. If they are in a situation with low personalization and low transparency, they see low benefits in particular, but due to their low DTVP, are less likely to investigate in detail which information might be collected. However, if low personalization is combined with high transparency, it gets obvious that a lot of information is collected about the individual. Thus, they might perceive this situation as unfair because the service only offers low personalization, but intrudes their privacy boundaries unnecessarily. If highly personalized services are offered, then individuals with low DTVP are particularly interested in the benefits and transparency features might even foster a feeling of fairness, as reciprocity (i.e. telling customers why their data is needed and how it will be used) has been shown to increase the probability of disclosing information and enhances users' perceptions of justice [12]. Thus, we hypothesize:

H2a: For individuals with low DTVP, the difference in information disclosure intentions between services with low and high transparency is significantly larger in the low personalization scenario than in the high personalization scenario.

On the contrary, individuals with high DTVP have a high inherent need to maintain their privacy boundaries [22]. Thus, in order to effectively manage their privacy, they are less willing to disclose information in general as they associate higher losses with information disclosure. Therefore, they are in general skeptical about information disclosure and have low intentions to do so, in particular for a low personalized service. When facing a highly personalized service, however, they also see some benefits. Yet, trying to establish a reciprocal relationship through transparency features might have counterproductive effects when dealing with individuals with high DTVP: It could even inflate their perceived privacy risks and lead to higher privacy concerns by strengthening their perception of boundary intrusion and fears of future boundary turbulences. We therefore hypothesize:

H2b: For individuals with high DTVP, the difference in disclosure intentions between services with low and high transparency is significantly larger in the high personalization scenario than in the low personalization scenario.

\section{Methodology}

We used an experimental setup to test our hypotheses. Next, we outline the experimental scenarios and procedure, the measurement of the constructs, and details on sampling and respondents.

\subsection{Experimental scenarios and procedure}

A quasi-experimental $2 \times 2$ between-subjects factorial design was chosen: Most important, it allowed us to design and control the independent variables of personalization and information transparency as well 
as to include several potential covariates, while it enabled at the same time the distribution of our experiment online within the natural internet environment for users of personalized services. Moreover, the scenario-based method facilitates the studying of future states while evaluating them from the respondents' contemporary perspectives [5].

To make our experiment as realistic as possible, the participants were presented with a cover story about a new online service which serves to support users in finding relevant events that match their individual preferences. According to the scenario, the new website was under construction and would be launched shortly. The participants were encouraged to evaluate the offered service based on website screen-shots. Later on, they were asked to complete a short questionnaire about their perception of the service.

Participants were randomly assigned to four distinct scenarios. As we employed a between-subject design, each subject was exposed to only one experimental condition. This between-subject design allowed us to avoid any carryover effects that are common for many within-subjects designs [33].

The experimental treatments were personalization (low versus high) and information transparency (low versus high). The distinct levels of personalization in this experiment were determined by the extent to which the service is able to find relevant events, provide recommendations, and tailor its newsletter to a customer's actual preferences and online behavior. In the low personalization condition a user was able to search events according to his or her tastes or browse in one of the proposed event categories. However, the high personalization treatment not only facilitated personalized search but also offered accurately tailored event recommendations, an individualized newsletter, and the option to integrate events into a user's personal calendar. The level of information transparency is reflected by the extent to which an online service provider offers specific features that enable customers to access the data collected about them (e.g. via their customer profile) as well as informs them on how and for what purposes the acquired information is going to be used. In the low transparency setting only the website's privacy policy was available to participants via a link provided below the website screenshot. With this manipulation we intended to reflect a realistic scenario that most users of online services are facing (status quo). In the high transparency condition, we provided users with an extra page "Customer Profile". It included explicit information about the purposes for which customer data was gathered and used and for how long it would be stored. It also highlighted the customer's right to oppose the processing of personal data for legitimate reasons. Finally, an overview of the personal information stored in the company's database was depicted. We chose particular pieces of information that a web site might legitimately ask for to offer personalized event recommendations.

After being confronted with the stimulus material, study participants first had to complete the manipulation check items. This was followed by an assessment of the participants' understanding of the proposed service offer with a control question, measures of DTVP, the respondents' overall experience with online personalization offerings, and previous encounters with event recommendation services as potential confounding variables. Then, the test subjects indicated their intentions to disclose information to the described service. Lastly, we collected demographic information and debriefed the participants on the study's actual background.

\subsection{Measurement of constructs}

We adapted constructs from measurement scales used in prior studies to fit the context of personalized event recommendations. Our survey questions are statement-like items that are rated on a 7-point Likert scale. We generally used at least three or four items per construct to adequately identify the construct and to assess its validity [34]. We used a short DTVP questionnaire [22,23] to measure individuals' privacy preferences. To measure the intention to disclose information (ID), we adapted the scale of Malhotra et al. [35]. We also included control variables such as gender, age, education, income level, internet use, and experience with the internet (EXPI) that were employed by previous studies [22,11,21,23]. In addition, we measured the participants' experience with online personalization [36] and whether or not they had used similar event recommendation services in the past (EXPP) [11,21]. Due to space limitations, we do not include the items in this paper. They can be obtained from the authors upon request.

\subsection{Sampling and participants}

We conducted a pilot test with 28 participants to check whether the developed experimental manipulations worked effectively. Moreover, the pretest was used to assess the clarity and conciseness of the instructions and items. All test subjects were also encouraged to give qualitative feedback. Their reviews were used to shorten the questionnaire and to improve the wording and layout of a few items.

The data for our main study was collected in April 2016. We conducted our scenario-based experiment online. This is an appropriate way to reach potential users of an event recommendation service because a 
regular online access is required to use such services. 295 German participants were recruited via e-mails, social networks, forums, and local online classified advertisings. To assure the high quality of our data set, we applied a data cleaning process to detect satisficing participants. We deleted answers with very low response time and respondents who failed to answer a control question [37]. 286 valid responses remained. In order to obtain a medium effect size $(f=.25)$, with a power of .80 at .05 significance level, the required total sample size is 128 . Thus, the size of the sample should be enough to observe medium effects. Demographics and descriptive statistics are presented in table 1 . We tested the distribution of gender, age, income, occupation, internet experience and experience with personalized services among our different groups and did not find any significant differences.

Table 1. Respondents' characteristics

\begin{tabular}{llr}
\hline Variable & Category & \multicolumn{1}{c}{$\mathbf{N}$} \\
\hline Gender & Female & $191(66.8 \%)$ \\
& Male & $95(33.2 \%)$ \\
\hline & $18-25$ & $118(41.3 \%)$ \\
Age group & $26-34$ & $89(31.1 \%)$ \\
& $35-44$ & $23(8 \%)$ \\
& $45-54$ & $36(12.6 \%)$ \\
& $55-64$ & $19(6.6 \%)$ \\
& 65 or more & $1(0.3 \%)$ \\
\hline & In training & $154(53.8 \%)$ \\
Occupation & Working & $119(41.6 \%)$ \\
& Not employed & $9(3.1 \%)$ \\
& Other & $4(1.4 \%)$ \\
\hline & Less than 1 & $24(8.4 \%)$ \\
& $1-2$ & $71(24.8 \%)$ \\
Internet usage & $3-4$ & $98(34.3 \%)$ \\
(hours per day) & $5-6$ & $53(18.5 \%)$ \\
& $7-10$ & $30(10.5 \%)$ \\
& 10 or more & $7(2.4 \%)$ \\
& Not specified & $3(1 \%)$ \\
\hline Experience with & Yes & $54(18.9 \%)$ \\
event recommen- & No & $232(81.1 \%)$ \\
dation services & & \\
\hline
\end{tabular}

\section{Findings}

In the following, we show that our manipulation was successful, discuss our measurement model validation, and present the results of our study.

\subsection{Manipulation check}

Before proceeding with the analysis of the differences in behavioral intentions among test groups, a manipulation check was carried out. The independent sample t-tests revealed that the study participants distinguished between different levels of personalization and information transparency across the designed conditions as expected. In the high personalization setting, respondents rated personalization as higher compared to the low personalization setting $(\mathrm{M} \Delta=-.38 ; \mathrm{t}(284)=-3.276$, $\mathrm{p}$ $<.005)$. Similarly, information transparency received higher scores in the high transparency conditions than in the low transparency conditions ( $\mathrm{M} \Delta=-1.02$; $\mathrm{t}(268.796)=-10.525, \mathrm{p}<.001)$.

Moreover, we wanted to further investigate the interaction between individuals DTVP, personalization, and transparency. As DTVP is a personality trait, we could not manipulate it but measured it via scales after the manipulation took place. To show that DTVP is not influenced by the manipulation, we conducted an ANOVA with DTVP as dependent and transparency and personalization as independent variables. As expected, we did not get any significant main or interaction effects. Thus, we split our sample in two groups with low vs. high DTVP to continue with our analysis.

\subsection{Measurement model validation}

Confirmatory factor analysis (CFA) was used to assess the validity and reliability of our latent variables DTVP, ID, EXPI and EXPP. We employed principal components analysis with Varimax rotation. We obtained four factors as expected with eigenvalues greater than one. A total of $82.36 \%$ of the variance can be explained by these four factors. Besides, we used the regression method to calculate factor scores which will be used for further analysis. A summary of the assessment is given in table 2 .

In order to assess the convergent validity of measured reflective constructs, we checked the factor loadings (all above 0.7), reliability of items (Cronbach's $\alpha$ exceeds 0.7 for all constructs), and average variance extracted (AVE; above 0.5 for all constructs so that the latent construct accounts for the majority of the variance of its indicators) $[34,38]$.

The discriminant validity of the measurement instrument was evaluated in two steps. First, we controlled if the items load more strongly on their corresponding construct than on other constructs in the model, which was fulfilled. This means that all constructs share more variance with their indicators than with other latent constructs. Second, we tested the fulfillment of the Fornell-Larcker criterion which suggests that the square root of the AVE for each variable should be greater than its correlation with any other construct in the model [39]. All latent variables 
Table 2. Statistics of latent constructs

\begin{tabular}{ccccccc}
\hline Constructs & $\begin{array}{c}\text { Items per } \\
\text { construct }\end{array}$ & $\begin{array}{c}\text { Factor } \\
\text { loadings }\end{array}$ & Mean & $\begin{array}{c}\text { Std. } \\
\text { Deviation }\end{array}$ & $\begin{array}{c}\text { Chron- } \\
\text { bach' s a }\end{array}$ & AVE \\
\hline DTVP & DTVP_01 & .883 & 4.47 & 1.68 & & \\
& DTVP_02 & .881 & 4.88 & 1.68 & .84 & .66 \\
& DTVP_03 & .769 & 4.72 & 1.76 & & \\
& DTVP_04 & .697 & 4.90 & 1.72 & & .86 \\
\hline ID & ID_01 & .934 & 4.02 & 1.66 & & \\
& ID_02 & .933 & 4.10 & 1.68 & .96 & .81 \\
& ID_03 & .923 & 4.04 & 1.66 & & \\
\hline EXPI & EXPI_01 & .920 & 5.78 & 1.23 & & .93 \\
& EXPI_02 & .899 & 5.68 & 1.26 & .93 & \\
\hline \multirow{2}{*}{ EXPP } & EXPI_03 & .887 & 5.74 & 1.24 & & \\
& EXPP_01 & .908 & 3.41 & 1.69 & & \\
& EXPP_02 & .883 & 3.47 & 1.73 & .92 & \\
& EXPP_03 & .870 & 3.47 & 1.72 & & \\
\hline
\end{tabular}

fulfilled this criterion. The correlation matrix for all latent constructs and their AVEs are given in table 3.

Thus, based on the results from the CFA we can conclude that developed measurement instrument fulfills the requirements of convergent and discriminant validity.

\section{Table 3. Correlations between latent constructs and AVE}

\begin{tabular}{cccccc}
\hline Constructs & AVE & EXPI & EXPP & DTVP & ID \\
\hline EXPI & .81 & $\mathbf{0 . 9}$ & & & \\
\hline EXPP & .79 & $.490^{\star *}$ & $\mathbf{0 . 8 9}$ & & \\
\hline DTVP & .66 & $-.170^{\star *}$ & $-.190^{\star *}$ & $\mathbf{0 . 8 1}$ & \\
\hline ID & .86 & $.135^{\star}$ & $.268^{\star *}$ & $-.349^{* *}$ & $\mathbf{0 . 9 3}$ \\
\hline
\end{tabular}

Note: The square root of the AVE for each construct is shown on the diagonal in bold font.

\subsection{Results}

To analyze our data, we conducted an ANCOVA with personalization, transparency, and DTVP as independent variable and intention to disclose as dependent variable after checking that all necessary assumptions of ANCOVA were fulfilled. With regard to potential covariates, we first included all control variables. Experience with personalization was the only significant covariate and was thus the only covariate included in our final model.

The results of ANCOVA showed a significant interaction effect between personalization and DTVP $(\mathrm{F}(1,275)=7.04, \mathrm{p}<.01)$, which is depicted in figure 1. To assess this interaction in more detail, we applied a post-hoc test to find out which groups differed from each other with regard to individuals' disclosure intentions. To prevent the loss of statistical power resulting from Bonferroni corrected estimates, a Sidak correction was used [40].

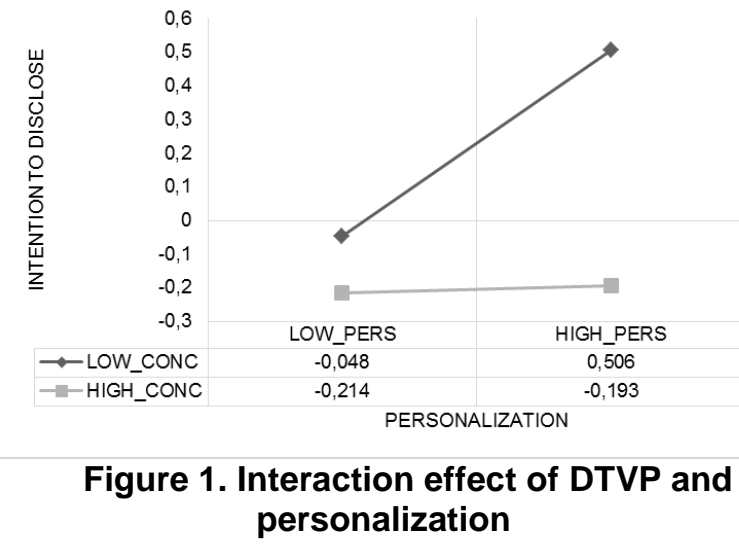

As a result, we can observe that subjects with low DTVP have higher intentions to disclose information to the highly personalized event recommendation service than those with high DTVP (mean difference 0.564, p $<0.05)$. Therefore, we find support for hypothesis $1 \mathrm{a}$. Furthermore, our results illustrate that individuals with low DTVP have higher intentions to disclose information to the highly personalized service compared to their disclosure intentions regarding the poorly personalized alternative, providing support for our hypothesis $1 \mathrm{~b}$ (mean difference $0.681, \mathrm{p}<0.05$ ).

However, no significant interaction was found between the distinct levels of personalization, transparency, and DTVP $(\mathrm{F}(1,275)=1.711, \mathrm{p}>.05)$. The differences in disclosure intentions between the services with low and high levels of personalization are not statistically significant in distinct levels of information transparency for both types of individuals either with low or high DTVP. Hence our hypotheses $2 \mathrm{a}$ and $2 \mathrm{~b}$ are not supported by the data.

Furthermore, the covariate experience with personalization was significantly related to the participants' intention to disclose information $(\mathrm{F}(1,275)=21.87, \mathrm{p}<.01)$. The $\beta$-value for the covariate was positive $(\beta=.264, \mathrm{t}=4.677, \mathrm{p}<.01)$ 
uncovering a positive relationship between experience with personalization and intention to disclose information to personalized services.

In addition, we tracked respondents' clicks on the provided privacy policy link which reflects their boundary management process to some extent [29]. Some individuals were apparently looking for privacy information to perform their risk-benefit calculus with regard to information disclosure to the offered service. The results show that only $16.8 \%$ of the respondents (48 subjects) clicked on the link that forwarded them to the website's privacy policy, while the majority $(83.2 \%)$ did not do so. Furthermore, the majority of clicks $(64.6 \%)$ was made by people with high DTVP (31 subjects). This is plausible because these people highly value their privacy and thus like to get more insights into personal data collection and usage before deciding whether to disclose information or not.

\section{Discussion}

The objective of our study was to investigate how the interaction between individuals' privacy dispositions, the personalization of a service, and information transparency influences the intention to disclose information. Our results show that the respondents' DTVP impacts their intention to disclose information to services with different levels of personalization. However, we did not find any significant effects with respect to transparency.

We contribute to theory by advancing the understanding of the role of individuals' privacy dispositions in information disclosure decisions in different situations. Individuals with low DTVP are more willing to trade their privacy for personalization benefits compared to individuals with high DTVP who do not appreciate information disclosure that much. The latter ones tend to protect their information boundaries from intrusions of highly personalized services and mostly avoid sharing required personal information in exchange for any benefits. In contrast, individuals with low DTVP are willing to open their boundaries and share their data with a firm to benefit from highly personalized services. Therefore, we also show that IBT is a very fruitful perspective to explain how an individual's personality, in particular an individuals' valuation of privacy, interacts with situational characteristics such as the level of personalization of a service. Third, we add further insights into the role of transparency to the knowledge base. Our hypotheses regarding the interaction between DTVP, personalization, and transparency were not supported by the data. Further analysis revealed that the main effect of transparency and the interaction effect between transparency and personalization were also insignificant. Thus, the inclusion of transparency features did not change individuals' behavioral intentions considerably. Nonetheless, we strongly encourage further research on this topic as our study was also not free from limitations, as discussed below, so that further evidence is needed. However, one additional explanation might be that transparency can evoke contrary effects: Although transparency features provide information which is relevant for rational decision making and increases the perception of fairness [12], at the same time the presentation of this information may arouse privacy concerns which results in concealing personal data [41]. Besides, previous research found that consumers respond positively to firms' implicit use of personalization (e.g. productbased e-mail recommendations). However, individuals' responses to explicit use of personalized greetings (e.g. a customer's name) were negative because such use of customer information compromised customer's anonymity and gave rise to privacy concerns [42]. Overall, the effect of transparency features needs further investigation.

Our study also has practical implications. Businesses that want to offer personalized services and thus need access to personal information might benefit from knowledge about the privacy dispositions of their customers. While people with low DTVP are willing to disclose personal information if they get benefits, people with high DTVP seem to be more skeptical and have lower intentions to disclose information. This makes it very difficult for such businesses to offer personalized services for this customer segment. Thus, organizations could either try to focus on attracting individuals with lower DTVP or to further investigate how individuals with higher DTVP could be persuaded to share the necessary information nonetheless.

We note several limitations of our study, which offer avenues for future research. The size of our convenience sample only allowed us to detect effects with a medium effect size, thus rerunning the experiment with more participants and a more representative sample might result in slightly different findings. In addition, a field experiment in a more authentic research setting (e.g. a real website or a mobile app) will likely provoke stronger perceptions of not only privacy risks regarding information transparency and information sharing but also of feelings of reciprocity and fairness. Moreover, we measured self-reported consumer intentions instead of actual behavior. Hence, our results do not account for the intention-behavior gap [43]. We had no possibility to measure the actual usage of online personalization in an authentic environment. Therefore, it would be worth-while to extend the scope of this research setting by testing our model on a real website or app. 


\section{Conclusion}

Our study shows that individuals' privacy dispositions influence their personalization-privacy trade-off. In particular, we found an interaction effect of personalization and DTVP: For people with low DTVP, the personalization of a service offering has a stronger effect on intentions to disclose information than for people with high DTVP. While we did not find any interaction between DTVP, personalization, and transparency, we hope that our findings and discussion inspire further research on this topic to gain more evidence on the effect of transparency features. Overall, digital businesses should consider taking into account their customers' privacy dispositions to be able to focus on the most promising customer segments only or to offer several levels of service personalization that match each customer's demands. This could be done by either assessing customers' privacy preferences, i.e. through short questionnaires, or by offering customers' the opportunity to self-select the appropriate level of service personalization for them, which is a promising avenue to increase not only customer satisfaction but also business success.

\section{References}

[1] S. Thirumalai and K.K. Sinha, "To Personalize or Not to Personalize Online Purchase Interactions: Implications of Self-Selection by Retailers." Information Systems Research, vol. 24 , no. 3, 2013, pp. 683-708.

[2] A. Jones, "Identity and marketing: Capturing, unifying, and using customer data to drive revenue growth | Insight VentureBeat." VentureBEat. Avaliable at: http://insight.venturebeat.com/report/customer-identitymarketing-capturing-storing-and-enriching-right-data, accessed on August 6, 2015.

[3] M.J. Culnan and P.K. Armstrong, "Information Privacy Concerns, Procedural Fairness, and Impersonal Trust: An Empirical Investigation.” Organization Science, vol. 10, no. 1, 1999, pp. 104-115.

[4] TRUSTe, "TRUSTe/NCSA US Consumer Privacy Index 2016." TRUSTe. Avaliable at:

https://www.truste.com/resources/privacy-research/ncsaconsumer-privacy-index-us/, accessed on June 12, 2016.

[5] H. Sheng, F.F.-H. Nah, and K. Siau, “An Experimental Study on Ubiquitous Commerce Adoption: Impact of Personalization and Privacy Concerns." Journal of the Association for Information Systems, vol. 9, no. 6, 2008, pp. 344-376.

[6] R.K. Chellappa and R.G. Sin, "Personalization versus Privacy: An Empirical Examination of the Online
Consumer's Dilemma." Information Technology and Management, vol. 6, no. 2-3, 2005, pp. 181-202.

[7] I.-H. Hann, K.-L. Hui, S.-Y.T. Lee, and I.P.L. Png, "Overcoming Online Information Privacy Concerns: An Information-Processing Theory Approach." Journal of Management Information Systems, vol. 24, no. 2, 2007, pp. 13-42.

[8] H. Treiblmaier and I. Pollach, "Users' Perceptions of Benefits and Costs of Personalization.” ICIS 2007

Proceedings, Montreal, Quebec, 2007.

[9] N.F. Awad and M.S. Krishnan, "The Personalization Privacy Paradox: An Empirical Evaluation of Information Transparency and the Willingness to Be Profiled Online for Personalization." MIS Quarterly, vol. 30, no. 1, 2006, pp. 13-28.

[10] S.Y. Ho, "Opportunities and challenges of mobile personalization: An exploratory study.” ECIS 2009

Proceedings, Verona, Italy, 2009.

[11] H. Xu, H.-H. Teo, B.C.Y. Tan, and R. Agarwal, "The Role of Push--Pull Technology in Privacy Calculus: The Case of Location-Based Services." Journal of Management Information Systems, vol. 26, no. 3, 2009, pp. 135-173.

[12] J.C. Zimmer, R. Arsal, M. Al-Marzouq, D. Moore, and V. Grover, "Knowing Your Customers: Using a Reciprocal Relationship to Enhance Voluntary Information Disclosure." Decision Support Systems, vol. 48, no. 2, 2010, pp. 395-406.

[13] J.Y. Tsai, S. Egelman, L. Cranor, and A. Acquisti, "The Effect of Online Privacy Information on Purchasing Behavior: An Experimental Study." Information Systems Research, vol. 22, no. 2, 2011, pp. 254-268.

[14] R.K. Chellappa and S. Shivendu, "An Economic Model of Privacy: A Property Rights Approach to Regulatory Choices for Online Personalization." Journal of Management Information Systems, vol. 24, no. 3, 2007, pp. 193-225.

[15] T.-P. Liang, H.-J. Lai, and Y.-C. Ku, "Personalized Content Recommendation and User Satisfaction: Theoretical Synthesis and Empirical Findings." Journal of Management Information Systems, vol. 23, no. 3, 2006, pp. 45-70.

[16] S.-J. Hong and K.Y. Tam, "Understanding the Adoption of Multipurpose Information Appliances: The Case of Mobile Data Services." Information Systems Research, vol. 17, no. 2, 2006, pp. 162-179.

[17] Westin, A.F., Privacy and freedom. Athenaum, New York, 1967.

[18] T. Dinev, P. Hart, and M.R. Mullen, "Internet privacy concerns and beliefs about government surveillance - An 
empirical investigation." The Journal of Strategic

Information Systems, vol. 17, no. 3, 2008, pp. 214-233.

[19] T. Dinev and P. Hart, "An Extended Privacy Calculus Model for E-Commerce Transactions." Information Systems Research, vol. 17, no. 1, 2006, pp. 61-80.

[20] H.J. Smith, T. Dinev, and H. Xu, "Information Privacy Research: An Interdisciplinary Review.” MIS Quarterly, vol. 35, no. 4, 2011, pp. 989-1016.

[21] T. Li and T. Unger, "Willing to pay for quality personalization? Trade-off between quality and privacy." European Journal of Information Systems, vol. 21, no. 6, 2012, pp. 621-642.

[22] H. Xu, T. Dinev, J. Smith, and P. Hart, "Information Privacy Concerns: Linking Individual Perceptions with Institutional Privacy Assurances." Journal of the Association for Information Systems, vol. 12, no. 12, 2011, pp. 798-824.

[23] Y. Li, "The impact of disposition to privacy, website reputation and website familiarity on information privacy concerns." Decision Support Systems, vol. 57, 2014, pp. 343 354.

[24] K.-L. Hui, H.H. Teo, and S.-Y.T. Lee, "The Value of Privacy Assurance: An Exploratory Field Experiment." MIS Quarterly, vol. 31, no. 1, 2007, pp. 19-33.

[25] Y. Li, "Theories in online information privacy research: A critical review and an integrated framework." Decision Support Systems, vol. 54, no. 1, 2012, pp. 471-481.

[26] Petronio, S., Boundaries of Privacy. State University of New York Press, Albany, NY, 2002.

[27] M.J. Culnan and R.J. Bies, "Consumer Privacy: Balancing Economic and Justice Considerations." Journal of Social Issues, vol. 59, no. 2, 2003, pp. 323-342.

[28] S. Petronio, "Communication Boundary Management: A Theoretical Model of Managing Disclosure of Private Information between Marital Couples." Communication Theory, vol. 1, no. 4, 1991, pp. 311-335.

[29] M.J. Metzger, "Communication Privacy Management in Electronic Commerce.” Journal of Computer-Mediated Communication, vol. 12, no. 2, 2007, pp. 335-361.

[30] J.T. Child, J.C. Pearson, and S. Petronio, "Blogging, communication, and privacy management: Development of the Blogging Privacy Management Measure." Journal of the American Society for Information Science and Technology, vol. 60, no. 10, 2009, pp. 2079-2094.

[31] J. Sutanto, E. Palme, Chuan-Hoo Tan, and Chee Wei Phang, "Addressing the Personalization-Privacy Paradox: An Empirical Assessment from a Field Experiment on
Smartphone Users.” MIS Quarterly, vol. 37, no. 4, 2013, pp. 1141-1164.

[32] S.Y.X. Komiak and I. Benbasat, "The Effects of Personalization and Familiarity on Trust and Adoption of Recommendation Agents." MIS Quarterly, vol. 30, no. 4, 2006, pp. 941-960.

[33] Field, A., G. Hole, How to Design and Report Experiments. Sage Publications Ltd., London, UK, 2002.

[34] Hair, J.F., W.C. Black, B.J. Babin, R.E. Anderson, Multivariate Data Analysis. Pearson, Upper Saddle River, NJ, 2009.

[35] N.K. Malhotra, S.S. Kim, and J. Agarwal, "Internet Users' Information Privacy Concerns (IUIPC): The Construct, the Scale, and a Causal Model." Information Systems Research, vol. 15, no. 4, 2004, pp. 336-355.

[36] R.T. Frambach, H.C.A. Roest, and T.V. Krishnan, "The impact of consumer Internet experience on channel preference and usage intentions across the different stages of the buying process." Journal of Interactive Marketing, vol. 21 , no. 2, 2007, pp. 26-41.

[37] N. Malhotra, "Completion Time and Response Order Effects in Web Surveys." Public Opinion Quarterly, vol. 72, no. 5, 2008, pp. 914-934.

[38] S.B. MacKenzie, P.M. Podsakoff, and N.P. Podsakoff, "Construct Measurement and Validation Procedures in MIS and Behavioral Research: Integrating New and Existing Techniques.” MIS Quarterly, vol. 35, no. 2, 2011, pp. 293 334.

[39] C. Fornell and D.F. Larcker, "Evaluating Structural Equation Models with Unobservable Variables and Measurement Error." Journal of Marketing Research, vol. 18 , no. 1,1981 , pp. $39-50$.

[40] Field, A., Discovering Statistics Using IBM SPSS Statistics. Sage Publications Ltd., London, 2013.

[41] L.K. John, A. Acquisti, and G. Loewenstein, "Strangers on a Plane: Context-Dependent Willingness to Divulge Sensitive Information." Journal of Consumer Research, vol. 37, no. 5, 2011, pp. 858-873.

[42] S. Wattal, R. Telang, T. Mukhopadhyay, and P. Boatwright, "What's in a "Name"? Impact of Use of Customer Information in E-Mail Advertisements." Information Systems Research, vol. 23, no. 3-NaN-1, 2011, pp. 679-697.

[43] P.A. Norberg, D.R. Horne, and D.A. Horne, "The Privacy Paradox: Personal Information Disclosure Intentions versus Behaviors." Journal of Consumer Affairs, vol. 41, no. 1, 2007, pp. 100-126. 